\title{
THE NUMBER OF FACTORIZATIONS OF NUMBERS LESS THAN $x$ INTO DIVISORS GREATER THAN $y$
}

BY

\author{
DOUGLAS HENSLEY
}

Abstract. Let $A(x, y)$ be the number in the title. There is a function $h:[0, \infty) \rightarrow$ $[0,2]$, decreasing and convex, with $h(0)=2$ and $\lim _{r \rightarrow x} h(r)=0$, such that if $r=\log y / \sqrt{\log x}$ then as $x \rightarrow \infty$ with $r$ fixed,

$$
A(x, y)=\frac{C(r) x \exp (h(r) \sqrt{\log x})}{(\log x)^{3 / 4}}\left(1+O(\log x)^{-1 / 4}\right) .
$$

The estimate is uniform on intervals $0<r \leqslant R_{0}$. As corollaries we have for $\log y=\theta(\log x)^{1 / 4}$,

$$
\lim _{x \rightarrow \infty} \frac{A(x, y)}{A(x, 1) / y}=e^{\theta^{2} / 2},
$$

and if $\log y=o(\log x)^{1 / 4}$ then $A(x, y) \approx A(x, 1) / y$.

1. Introduction. In counting factorizations we make no distinction between $2 \cdot 2 \cdot 3$, $2 \cdot 3 \cdot 2$ and $3 \cdot 2 \cdot 2$, and list four factorizations of $12: 12,6 \cdot 2,4 \cdot 3$ and $3 \cdot 2 \cdot 2$.

Let $a(n)$ denote the number of such factorizations of $n$. MacMahon observed, about 1920 , that $\sum_{n=1}^{\infty} a(n) n^{-s}=\prod_{d=2}^{\infty}\left(1-d^{-s}\right)^{-1}$. Shortly thereafter Oppenheim considered the average and maximum values of $a(n), 1 \leqslant n \leqslant x$. He found

$$
A(x):=\sum_{n=1}^{x} a(n) \cong x \exp (2 \sqrt{\log x}) /\left(2 \sqrt{\pi}(\log x)^{3 / 4}\right),
$$

as did Szekeres and Turan somewhat later [2, 3]. Recently the question of the number of factorizations of numbers $n \leqslant x$ using only divisors $d \leqslant y$ was discussed, and estimated to within a factor of $(\log x)^{O(1)}[\mathbf{1}]$. Here we are concerned with

$$
A(x, y):=\sum_{n=1}^{[x]} a_{y}(n)
$$

where $a_{y}(n)$ is the number of factorizations of $n$ into divisors $d>y$. We make the conventional assumption that $a_{y}(1)=1$ for any $y$, so that $A(x, y)=0$ for $x<1$, and $A(x, y)=1$ for $1 \leqslant x \leqslant y$.

We prove the following results.

Received by the editors February 21, 1983.

1980 Mathematics Subject Classification. Primary 10H25; Secondary 60F10.

Key words and phrases. Complex analytic methods, elementary probability methods, large deviations. 
TheOrem 1. There exists $C_{1}>0$ such that if $y$ is an integer, $0<\varepsilon \leqslant 1$ and $1 \leqslant \log y \leqslant \varepsilon(\log x)^{1 / 4}$ then

$$
y A(x, y) / A(x) \in\left(1-C_{1} \varepsilon^{2}, 1+C_{1} \varepsilon^{2}\right) .
$$

To state Theorem 2 we need some notation. For $x>y>1$ let

$$
r=r(x, y)=\log y / \sqrt{\log x} .
$$

Let $q=q(r)$ be the unique solution in $0<q<1$ of

$$
2 \log q-\log (1-r q)+r q /(1-r q)=0 .
$$

REMARK. In this paper, $r$ is the reciprocal of the $r$ used in [1].

Let $h(r)=2 q+r q^{2} /(1-r q)$. Let $C(r)=\left\{4 \pi\left(1-r q+\frac{1}{2} r^{2} q^{2}\right)\right\}^{-1 / 2}$.

THEOREM 2. Uniformly in $(\log x)^{1 / 5} \leqslant \log y \leqslant R(\log x)^{1 / 2}$,

$$
A(x, y)=C(r) x(\log x)^{-3 / 4} e^{h(r) \sqrt{\log x}}\left(1+O_{R}\left(1 /(\log x)^{1 / 4}\right)\right),
$$

for any fixed $R>0$.

COROLlaRY. For arbitrary real $\theta>0$, with $\log y=\theta(\log x)^{1 / 4}$,

$$
\lim _{x \rightarrow \infty} y A(x, y) / A(x)=e^{\theta^{2} / 2} .
$$

REMARK. This explains the $1+O\left(\varepsilon^{2}\right)$ when $\log y \leqslant \varepsilon(\log x)^{1 / 4}$ in Theorem 1 ; no better result is possible.

The proof of Theorem 1 is an exercise in complex analysis and presents no great novelty. For the most part it follows Oppenheim's original proof.

The proof of Theorem 2 uses some of the techniques of [1]. The novelty here is that for the case at hand we can dispense with grouping factorizations according to the number of divisors from various intervals $\left(\alpha^{i-1}, \alpha^{i}\right)$, and we can evaluate explicitly the probabilities that arise in a temporary reformulation of the problem as a question of chance. This permits accuracy to within a factor of $1+O(\log x)^{-1 / 4}$ instead of $(\log x)^{O(1)}$ which we got in [1]. I think the new techniques might succeed with the old $K(x, y)$ and give similar improvements in accuracy. The hard step will be to evaluate more accurately the probabilities that arise in the $K(x, y)$ reformulation. $(K(x, y)$ is the number of factorizations using divisors $\leqslant y$.)

2. Complex analysis. Before we get into the matter too deeply we note that for fixed $y \in \mathbf{Z}$,

$$
\lim _{x \rightarrow \infty} A(x, y) / A(x)=1 / y
$$

by an elementary inclusion-exclusion argument based on (1.1) and the fact that $\prod_{d=2}^{y}(1-1 / d)=1 / y$.

To start the analysis now, let $f_{y}(s)=\prod_{n=y+1}^{\infty}\left(1-n^{-s}\right)^{-1}$, for $s=\sigma+i t, \sigma>1$. Then $f_{y}(s)=\sum_{n=1}^{\infty} a_{y}(n) / n^{s}$ for $\sigma>1$, where $a_{y}(n)$ is, as in $\S 1$, the number of ways in which $n$ is the product of integers greater than $y$.

Let $B(x, y)=\Sigma_{n \leqslant x}(x-n) a_{y}(n)$, and recall $A(x, y)=\Sigma_{n \leqslant x} a_{y}(n)$. 
As in Oppenhiem [2] let $f(s)=\prod_{n=2}^{\infty}\left(1-n^{-s}\right)^{-1}(\sigma>1)$ and let

$$
g_{y}(s)=\prod_{n=2}^{y}\left(1-n^{-s}\right) \text {. }
$$

Then $f_{y}(s)=f(s) g_{y}(s)$.

We have from [2] that there is an expansion

$$
f(s)=\exp (1 /(s-1))\left(1+\sum_{n=1}^{\infty} \alpha_{n}(s-1)^{n}\right),
$$

valid for $|s-1|<1 / 2$, and that $f(s)=O\left(|t|^{\varepsilon}\right)$ for any positive $\varepsilon$, uniformly for $|t| \geqslant t_{0}(\varepsilon)$ and $\sigma>1$.

We require an estimate for the growth of the coefficients $C_{k}(y)$ in the expansion

$$
\begin{aligned}
g_{y}\left(1+\frac{1}{w}\right) & =\frac{1}{y} \prod_{n=2}^{y}\left(1-\left(1-n^{-1 / w}\right) /(n-1)\right) \\
& =\frac{1}{y}\left(1+\sum_{k=1}^{\infty} C_{k}(y) w^{-k}\right) .
\end{aligned}
$$

Lemma 2.1. For $k \leqslant y,\left|C_{k}(y)\right| \leqslant(4 \log y)^{2 k}$, while $\left|C_{k}(y)\right| \leqslant(4 \log y)^{2 y}(k \geqslant y)$.

Proof. Expanding the product in (2.2) using $n^{-1 / w}=\sum_{j=0}^{\infty}(-1)^{j}(j !)^{-1}(\log n / w)^{j}$, we get

$$
C_{k}(y)=\sum_{\bar{V} \in S(k)}(-1)^{k} \prod_{n=2}^{y}(n-1)^{-\beta_{n}(\bar{V})}(\log n)^{V_{n}} / V_{n} !
$$

where

$$
S(k)=\left\{\bar{V}=\left(V_{2}, V_{3}, \ldots, V_{y}\right) \mid V_{2}, \ldots, V_{y} \geqslant 0 \in Z \text { and } \sum_{2}^{y} V_{n}=k\right\},
$$

and where $\beta_{n}(\bar{V})=1$ if $V_{n} \geqslant 1$, else 0 . Thus

$$
\left|C_{k}(y)\right| \leqslant(\log y)^{k} \sum_{\bar{V} \in S(k)} \prod_{n=2}^{y}\left(\frac{1}{n-1}\right)^{\beta_{n}(\bar{V})} \frac{1}{V_{n} !} .
$$

For any fixed $T \subseteq\{2,3, \ldots, y\}$ with $\# T=r \leqslant k$, the sum over the terms of (2.4) in which $V_{n} \geqslant 1$ if and only if $n \in T$ is

$$
\prod_{n \in T}(n-1)^{-1} \sum_{P(k, r)} \prod_{j=1}^{r} 1 / p_{j} !
$$

where $P(k, r)$ is the set of ordered partitions of $k$ into positive integers $p_{1}, p_{2}, \ldots, p_{r}$, and this

$$
=\prod_{n \in T}(n-1)^{-1} r^{k} / k !=\left(r^{k} / k !\right) \prod_{n \in T}(n-1)^{-1}
$$


Summing over all $T$ with $r$ elements, we get

$$
\begin{aligned}
\left(r^{k} / k !\right) \sum_{n_{1} \neq n_{2} \neq \cdots \neq n_{r}} \prod\left(n_{i}-1\right)^{-1} & \leqslant\left(r^{k} / k !\right)\left(\sum_{2}^{y}(n-1)^{-1}\right)^{r} \\
& \leqslant\left(r^{k} / k !\right)(1+\log y)^{r} .
\end{aligned}
$$

Summing over the possible values of $r$ gives $\sum_{r=1}^{\min \{y \cdot k\}}\left(r^{k} / k !\right)(1+\log y)^{r}$. For $k \leqslant y$ this yields

$$
\left|C_{k}(y)\right| \leqslant \frac{k^{k}}{(k-1) !}(1+\log y)^{k}(\log y)^{k} \quad \text { and } \quad\left|C_{k}(y)\right| \leqslant(4 \log y)^{2 k}
$$

while for $k>y,\left|C_{k}(y)\right| \leqslant(4 \log y)^{2 y}$.

After this we can copy [2]. We have

$$
B(x, y)=\sum_{n \leqslant x}(x-n) a_{y}(n)=\frac{1}{2 \pi i} \int_{2-i \infty}^{2+i \infty} \frac{x^{s+1}}{s(s+1)} f(s) g_{y}(s) d s .
$$

From the definition of $g_{y}(s)$ clearly $\left|g_{y}(s)\right| \leqslant y$ for $\sigma=1$. Thus in analogy with [2],

$$
B(x, y)=J(x, y)+O\left(x^{2} y\right), \quad \text { where } J(x, y)=\frac{1}{2 \pi i} \int \frac{x^{s+1}}{s(s+1)} f(s) g_{y}(s) d s
$$

and $\Gamma$ half-encircles 1 to the right at radius $a>0$. Further, by Cauchy's theorem, for $b>0$

$$
J(x, y)=\frac{1}{2 \pi i} \int_{b-i}^{b+i} \frac{x^{2+1 / w}}{(w+1)(2 w+1)} f_{y}(1+1 / w) d w+O\left(x^{2} y\right),
$$

on the change of variable $s=1+1 / w$. Now

$$
\frac{f(1+1 / w)}{(w+1)(2 w+1)}=\frac{1}{2} e^{w} \sum_{n=0}^{\infty} \delta_{n} w^{-2-n}
$$

uniformly on $\sigma=b$, with $\delta_{0}=1$ and $\delta_{n}$ certain constants, with $\left|\delta_{n}\right| \leqslant K^{n}$ for some $K>2$ and all $n[2]$.

If we set $C_{0}(y)=1$ we have

$$
\frac{f(1+1 / w) g_{y}(1+1 / w)}{(w+1)(2 w+1)}=\frac{e^{n}}{2 y} \sum_{n=0}^{\infty} \sum_{j=0}^{n} \delta_{n-j} C_{j}(y) w^{-n-2} .
$$

Let $d_{n}(y)=\sum_{j=0}^{n} \delta_{n-j} C_{j}(y)$. Then by Lemma 2.1, $d_{0}(y)=1$, and for log $y \geqslant 2 K$,

$$
\begin{aligned}
& \left|d_{n}(y)\right| \leqslant 2(4 \log y)^{2 n} \quad \text { for } n \leqslant y, \\
& \left|d_{n}(y)\right| \leqslant 2 K^{n-y}(4 \log y)^{2 y} \quad \text { for } n \geqslant y .
\end{aligned}
$$

As in [2] we now have

$$
\begin{aligned}
J(x, y)+O\left(x^{2} y\right)= & \frac{1}{2} \frac{x^{2}}{y \sqrt{\log x}} I_{1}(2 \sqrt{\log x}) \\
& +\frac{1}{2 \pi i} \int_{b-i \infty}^{b+i \infty} \frac{1}{2} \frac{x^{2}}{y} e^{w+\left(\log x / w^{\prime}\right)} \sum_{\nu=1}^{\infty} \frac{d_{\nu}(y)}{w^{\nu}} \frac{d w}{w^{2}} .
\end{aligned}
$$


If we take $b=\sqrt{\log x}\left(\geqslant 32 K(\log y)^{2}\right.$ by assumption $)$ and let $w=b+i t$ then

$$
\left|\sum_{\nu=1}^{\infty} \frac{d_{\nu}(y)}{w^{\nu}}\right|=O\left((\log y)^{2} /|w|\right) \quad \text { on } w=b+i t
$$

so the absolute value of the integral in (2.11) is

$$
\begin{aligned}
O\left(x^{2}(\log y)^{2} / y\right) \int_{0}^{\infty}|w|^{-3} \exp \left(b+b \log x /\left(b^{2}+t^{2}\right)\right) d t \\
=O\left(x^{2} e^{2 \sqrt{\log x}}(\log y)^{2} /(y \log x)\right) \\
\quad \cdot \int_{0}^{\infty}\left(1+h^{2}\right)^{-3 / 2} \exp \left(-h^{2} \sqrt{\log x} /\left(1+h^{2}\right)\right) d h
\end{aligned}
$$

with $h=t / \sqrt{\log x}=O\left(x^{2} e^{2 \sqrt{\log x}}(\log y)^{2} /\left(y(\log x)^{5 / 4}\right)\right)$. Thus

$$
\begin{aligned}
J(x, y)+O\left(x^{2} y\right)= & \frac{1}{2} \frac{x^{2}}{y \sqrt{\log x}} I_{1}(2 \sqrt{\log x}) \\
& +O\left(x^{2} e^{2 \sqrt{\log x}}(\log y)^{2} /\left(y(\log x)^{5 / 4}\right)\right),
\end{aligned}
$$

and since $I_{1}(z)=(2 \pi z)^{-1 / 2} e^{z}(1+O(1 / z))$,

$$
B(x, y)=\frac{x^{2} e^{2 \sqrt{\log x}}}{4 \sqrt{\pi} y(\log x)^{3 / 4}}\left(1+O\left((\log y)^{2} / \sqrt{\log x}\right)\right),
$$

uniformly in $(2 K)^{2} \leqslant(\log y)^{2} \leqslant \sqrt{\log x} /(32 K)$.

Now

$$
\frac{\partial J}{\partial x}=\frac{1}{2 \pi i} \int_{C} \frac{x^{1+1 / w}}{w(w+1)} f_{y}(1+1 / w) d w
$$

and

$$
\frac{\partial^{2} J}{\partial x^{2}}=\frac{1}{2 \pi i} \int_{C} \frac{x^{1 / w}}{w^{2}} f_{y}(1+1 / w) d w
$$

where $C$ is the half circle to the right of $O$ about $O$ at radius $1 / a$.

Expanding $f_{v}(1+1 / w) e^{-w} / w(w+1)$ as a series in $1 / w$ gives

$$
\frac{\partial J}{\partial x}=\frac{x}{y} \frac{e^{2 \sqrt{\log x}}}{2 \sqrt{\pi}(\log x)^{3 / 4}}\left\{1+O\left(\frac{(\log y)^{2}}{\sqrt{\log x}}\right)\right\} \quad \text { and } \quad \frac{\partial^{2} J}{\partial x^{2}}=O\left(\frac{1}{y} \frac{e^{2 \sqrt{\log x}}}{(\log x)^{3 / 4}}\right) .
$$

both uniformly in $(2 K)^{2} \leqslant(\log y)^{2} \leqslant(32 K)^{-1} \sqrt{\log x}$. The details parallel those of (2.7)-(2.12).

Finally for this range of $y$ by an exact copy of the Tauberian argument in [2] from $B(x) \sim J(x)$ to $A(x) \sim J^{\prime}(x)$ we have

$$
\begin{aligned}
A(x, y) & =J^{\prime}(x, y)+O\left(x e^{(1+1 / 2 K) \sqrt{\log x}}\right) \\
& =\frac{x}{y} \frac{e^{2 \sqrt{\log x}}}{2 \sqrt{\pi}(\log x)^{3 / 4}}\left(1+O\left(\frac{(\log y)^{2}}{\sqrt{\log x}}\right)\right),
\end{aligned}
$$

which proves Theorem 1. 
3. Elementary methods. Our proof of Theorem 2 is based on a counting principle set in the language of probability, and on the calculus that boils down the resulting estimate of $A(x, y)$ to the form $C(r) x \log ^{-3 / 4} x e^{h(r) \sqrt{\log x}}\left(1+O_{R}\left(\log ^{-1 / 4} x\right)\right)$. The devices used here seem to be considerably stronger than the standard complex-analytic approach of $\$ 2$. Indeed one purpose of $\S 2$, aside from treating the case when $y$ is too small for the approach of this section, is to highlight the advantage here of real methods.

We start with a reformulation of $A(x, y)$.

$$
A(x, y)=\#\left\{\xi:\{y+1, y+2, \ldots, x\} \rightarrow\{0,1,2, \ldots\} \mid \sum_{d>y} \xi_{d} \log d \leqslant \log x\right\} .
$$

Let $u=\log x / \log y$ and $r=\log y / \sqrt{\log x}=\sqrt{\log x} / u$. Let $X_{1}, X_{2}, \ldots$ be independent random variables uniformly distributed on $\{y+1, \ldots, x\}$. For $n \leqslant u$, let $\bar{x}$ denote an $n$-tuple of integers. Then

$$
\begin{aligned}
& \operatorname{Prob}\left(\sum_{1}^{n} \log X_{i} \leqslant \log x\right) \\
& \quad=(x-y)^{-n} \#\left\{\bar{x} \mid y<x_{i} \leqslant x \text { for } 1 \leqslant i \leqslant n \text { and } \sum_{1}^{n} \log x_{i} \leqslant \log x\right\} .
\end{aligned}
$$

Associate with each $\bar{x}$ the $(x-y)$-tuple $\xi=\left(\xi_{y+1}, \xi_{y+2}, \ldots, \xi_{x}\right)$ with $\xi_{d}=$ the number of times $d$ occurs in $\bar{x}$. Then $\sum_{y+1}^{x} \xi_{d}=n$.

The number of times a given $\xi$ occurs among the set of all $n$-tuples $\bar{x}$ is $n ! \prod_{d=y+1}^{x}\left(\xi_{d} !\right)^{-1}$.

Let $\|\xi\|=\prod_{y+1}^{x} d^{\xi_{d}}$, and $\sigma(\xi)=\sum_{y+1}^{x} \xi_{d}$. Then

$$
\operatorname{Prob}\left(\sum_{1}^{n} \log X_{i} \leqslant \log x\right)=(x-y)^{-n} n ! \sum_{\|\xi\| \leqslant x, \sigma(\xi)=n} \prod_{y+1}^{x}\left(\xi_{d} !\right)^{-1},
$$

and

$$
\sum_{\|\xi\| \leqslant x, \sigma(\xi)=n} \prod_{y+1}^{x}\left(\xi_{d} !\right)^{-1}=(x-y)^{n}(n !)^{-1} \operatorname{Prob}\left(\sum_{1}^{n} \log X_{i} \leqslant \log x\right) .
$$

Now $A(x, y)=\Sigma_{\|\xi\| \leqslant x} 1$, so with the notation $P_{n}=\operatorname{Prob}\left(\sum_{1}^{n} \log X_{i} \leqslant \log x\right)$,

$$
A(x, y) \geqslant \sum_{n \leqslant u}(x-y)^{n}(n !)^{-1} P_{n} .
$$

The inequality is quite sharp, for, in fact, also

$$
A(x, y) \leqslant(1-2 / y)^{-1} \sum_{n \leqslant u}(x-y)^{n}(n !)^{-1} P_{n} .
$$

For the proof we need a lemma.

LEMMA 3.1. For any integer $t \geqslant 1$ and any real $s \geqslant t, 2 A(s, t) \leqslant A(2 s, t)$.

Proof. For $t>1, A(s, t-1)=\sum_{m=0}^{\infty} A\left(s / t^{m}, t\right)$. Also $A(s, t)=0$ for $s<1,1$ for $1 \leqslant s \leqslant t$, and $s-t+1$ for $\sqrt{s} \leqslant t \leqslant s$. Fix $T>1$ and $s \leqslant T$, and make the 
inductive assumption that $2 A(p, q) \leqslant A(2 p, q)$ for $p \leqslant T$ and $q \geqslant s$. This is clear if $s=T$. Let $M=[\log T / \log s]$. Then for $p<s$, evidently

$$
A(2 p, s-1)-2 A(p, s-1) \geqslant 0,
$$

while for $T \geqslant p \geqslant s$,

$$
\begin{aligned}
A(2 p, s-1)-2 A(p, s-1) \geqslant & \sum_{m=0}^{M} A\left(2 p / s^{m}, s\right)-2 A\left(p / s^{m}, s\right) \\
\geqslant & (1-2)+\left(\left(2 p / s^{M-1}-s+1\right)-2\left(p / s^{M-1}-s+1\right)\right) \\
& +\sum_{m=0}^{M-2}\left(A\left(2 p / s^{m}, s\right)-2 A\left(p / s^{m}, s\right)\right) \\
\geqslant & -1+(s-1)+0
\end{aligned}
$$

by the inductive assumption, $\geqslant 0$ for $s-1 \geqslant 1$.

We now continue with the proof of (3.6). Let

$$
A_{1}(x, y)=\#\left\{\xi \mid\|\xi\| \leqslant x \text { and } \xi_{d}=0 \text { or } 1 \text { for all } d\right\},
$$

and let $A_{2}(x, y)=A(x, y)-A_{1}(x, y)$. Then $A_{2}(x, y) \leqslant \Sigma_{d>y} A\left(x / d^{2}, y\right)$. From Lemma 3.1 applied repeatedly, $A\left(x / d^{2}, y\right) \leqslant\left(2 / d^{2}\right) A(x, y)$. Thus

$$
\sum_{d>y} A\left(x / d^{2}, y\right) \leqslant 2 A(x, y) \sum_{d>y} 1 / d^{2}<(2 / y) A(x, y) .
$$

Let $H(x, y)=\sum_{\|\xi\| \leqslant x} \Pi_{y+1}^{x}\left(\xi_{d} !\right)^{-1}$ and let $H_{1}(x, y)$ be the same sum taken only over $\xi$ counted in $A_{1}(x, y)$. Clearly

$$
1 \geqslant H_{1}(x, y) / H(x, y) \geqslant A_{1}(x, y) / A(x, y) \text {. }
$$

Also $H_{1}(x, y)=A_{1}(x, y)$. Since $A_{1}(x, y) / A(x, y)>(1-2 / y)$ by (3.7) we conclude that

$$
A(x, y) \leqslant(1-2 / y)^{-1} H(x, y)=(1-2 / y)^{-1} \sum_{n \leqslant u}(x-y)^{n}(n !)^{-1} P_{n}
$$

which proves (3.6).

In view of (3.5) and (3.6) any estimate of $\Sigma_{n \leqslant u}(x-y)^{n}(n !)^{-1} P_{n}$ gives an estimate of $A(x, y)$.

For an upper bound we replace $X_{i}$ in $P_{n}=\operatorname{Prob}\left(\sum_{1}^{n} \log X_{i} \leqslant \log x\right)$ with random variables $Y_{i}$ independent and uniformly distributed on the real interval $(y, x)$, and set $P_{n}^{+}=\operatorname{Prob}\left(\sum_{1}^{n} \log Y_{i} \leqslant \log x\right) \geqslant P_{n}$. The probability density function for $\log Y_{i}$ has the form $e^{C t+D} I(\log y, \log x)$ and the convolution of $n$ copies has the form $e^{C t+D^{\prime}} S(t)$, where $S(t)$ is a spline which is zero for $t \leqslant n \log y$ and is $D^{\prime \prime}(t-n \log y)^{n}$ for $n \log y<t \leqslant \log x$. Explicit calculation now gives

$$
P_{n}^{+}=\left(\frac{\log x-\log y}{x-y}\right)^{n} \frac{x}{(n-1) !} \int_{0}^{1-(n-1) /(u-1)} h^{+}(t) d t,
$$

where

and

$$
h^{+}(t)=t^{n-1} \exp (-C(1-(n-1) /(u-1)-t))
$$

$$
C=(\log x-n \log y) /(1-(n-1) /(u-1)) .
$$


Let $I_{n}^{+}$be the definite integral in (3.8). Now with random variables $Z_{i}$ independent and uniformly distributed on $(y+1, x)$, we put

$$
P_{n}^{-}=\left(1-(x-y)^{-1}\right)^{n} \operatorname{Prob}\left(\sum_{1}^{n} \log Z_{i} \leqslant \log x\right) \leqslant P_{n},
$$

and calculate in the same way

$$
P_{n}^{-}=(\log x-\log (y+1))^{n}(x-y-1)^{-n}\left(x /(n-1) !\left(1-(x-y)^{-1}\right)^{n}\right) I_{n}^{-},
$$

where

$$
\begin{aligned}
& I_{n}^{-}=\int_{0}^{1-(n-1) /(v-1)} h^{-}(t), \quad v=\log x / \log (y+1), \\
& K=(\log x-n \log (y+1))(1-(n-1) /(v-1))^{-1}
\end{aligned}
$$

and

$$
h^{-}(t)=t^{n-1} \exp (-K(1-(n-1) /(v-1)-t)) .
$$

This simplifies to

$$
P_{n}^{-}=\left(\frac{\log x-\log (y+1)}{x-y}\right)^{n} \frac{x}{(n-1) !} I_{n}^{-} .
$$

Next we have a lemma about $I_{n}^{+}$and $I_{n}^{-}$.

LEMMA 3.2. Uniformly in integers $n \geqslant 2$ and in real $\theta \geqslant 0$,

$$
\int_{0}^{1} s^{n-1} e^{n \theta s-n \theta} d s=\frac{1}{n+n \theta}\left(1+O\left(\log ^{2} n / n\right)\right) .
$$

Proof. Since $e^{s-1}-\frac{1}{2}(s-1)^{2} \leqslant s \leqslant e^{s-1}$ for $0 \leqslant s \leqslant 1$,

$$
\begin{aligned}
\int_{0}^{1} s^{n-1} e^{n \theta(s-1)} d s & \leqslant \int_{0}^{1} e^{(n-1)(s-1)+n \theta(s-1)} d s \leqslant \int_{-\infty}^{1} e^{(n+n \theta-1)(s-1)} d s \\
& =\frac{1}{n(1+\theta)-1}=\frac{1}{n+n \theta}\left(1+O\left(\log ^{2} n / n\right)\right)
\end{aligned}
$$

Also

$$
\begin{aligned}
\int_{0}^{1} s^{n-1} e^{n \theta(s-1)} d s & \geqslant \int_{1-\log n / n}^{1} \exp \left(s-1-\log ^{2} n / 2 n^{2}\right)(n-1)+\theta n(s-1) d s \\
& \geqslant \int_{-\log n / n}^{0} \exp \left(n(1+\theta) r-r-\frac{1}{2}\left(\log ^{2} n / n\right)\right) d r \\
& \geqslant\left(1-\frac{1}{2} \log ^{2} n / n\right)\left(\frac{1}{n+n \theta-1}\right)\left(1-e^{-(1+\theta) \log n}\right) \\
& =\frac{1}{n+n \theta}\left(1+O\left(\log ^{2} n / n\right)\right) .
\end{aligned}
$$

Now

$$
I_{n}^{+}=\left(1-\frac{n-1}{u-1}\right)^{n}\left(\frac{1}{n+\log x-n \log y}\right)\left(1+O\left(\log ^{2} n / n\right)\right)
$$


by Lemma 3.2, while

$$
I_{n}^{-}=\left(1-\frac{n-1}{v-1}\right)^{n}\left(\frac{1}{n+\log x-n \log (y+1)}\right)\left(1+O\left(\log ^{2} n / n\right)\right) .
$$

Thus

$$
\begin{aligned}
P_{n}^{+}= & \left(\frac{\log x-\log y}{x-y}\right)^{n} \frac{x}{(n-1) !}\left(1-\frac{n-1}{u-1}\right)^{n} \\
& \cdot\left(\frac{1}{n+\log x-n \log y}\right)\left(1+O\left(\log ^{2} n / n\right)\right)
\end{aligned}
$$

and on simplification,

$$
P_{n}^{+}=\frac{(\log x)^{n}}{(x-y)^{n}} \frac{x}{(n-1) !}\left(1-\frac{n}{u}\right)^{n}\left(\frac{1}{n+\log x-n \log y}\right)\left(1+O\left(\log ^{2} n / n\right)\right) .
$$

Similarly

$$
P_{n}^{-}=\frac{(\log x)^{n}}{(x-y)^{n}} \frac{x}{(n-1) !}\left(1-\frac{n}{v}\right)^{n}\left(\frac{1}{n+\log x-n \log (y+1)}\right)\left(1+O\left(\log ^{2} n / n\right)\right) .
$$

We use (3.11) in (3.6) and (3.12) in (3.5) and conclude

$$
\begin{aligned}
A(x, y) \leqslant & \left(1-\frac{2}{y}\right)^{-1} \times \sum_{n<u} \frac{\log ^{n-1} x}{(n-1) ! n !}\left(1-\frac{n}{u}\right)^{n-1}\left(1-\frac{n}{\log x-n \log y+n}\right) \\
& \cdot\left(1+O\left(\log ^{2} n / n\right)\right)
\end{aligned}
$$

and

$$
\begin{aligned}
A(x, y) \geqslant & x \sum_{n<v} \frac{\log ^{n-1} x}{(n-1) ! n !}\left(1-\frac{n}{v}\right)^{n-1} \\
& \cdot\left(1-\frac{n}{\log x-n \log (y+1)+n}\right)\left(1+O\left(\log ^{2} n / n\right)\right) .
\end{aligned}
$$

We now claim that for $\sqrt{u}<n<u-\sqrt{u}$, the corresponding terms of (3.13) and (3.14) are in a ratio of $1+O(1 / \sqrt{\log x})$.

For

$$
(1-n / v)^{n-1}(1-n / u)^{1-n} \geqslant\left(1-u^{3 / 2}(1 / v-1 / u)\right)^{n-1} \geqslant\left(1-u^{1 / 2} /(y \log y)\right)^{u} \text {. }
$$

By assumption $\log y \geqslant \log ^{1 / 5} x$, so

$$
\left(1-u^{1 / 2} /(y \log y)\right)^{u} \geqslant\left(1-\log ^{1 / 5} x \exp \left(-\log ^{1 / 5} x\right)\right)^{u} \geqslant 1+O(1 / \sqrt{\log x}) .
$$

Also if $n<u-\sqrt{u}, \log x-n \log y \geqslant u^{-1 / 2} \log x$, so

$$
\begin{aligned}
(1- & \left.n(\log x-n \log (y+1)+n)^{-1}\right)\left(1-n(\log x-n \log y+n)^{-1}\right)^{-1} \\
= & 1+O\left(u^{1 / 2} / y \log x\right)=1+O(1 / \sqrt{\log x}) .
\end{aligned}
$$

This proves the claim. 
To dispose of the terms in (3.13) and (3.14) with $n \leqslant \sqrt{u}$ or $n \geqslant u-\sqrt{u}$, and for further use, we need another lemma.

LEMMA 3.3. Suppose $\left(C_{n}\right)$ is a log-concave sequence of positive numbers, with $C_{0}$ the largest. For each $\varepsilon>0$ let $N(\varepsilon)$ denote the largest $n$ with $C_{n}>\varepsilon C_{0}$, and let $m(\varepsilon)$ be the least $m$ with $C_{m}>\varepsilon C_{0}$. Then for $\varepsilon<1, \sum_{m(\varepsilon)}^{n(\varepsilon)} C_{k} \geqslant(1-2 \varepsilon) \sum_{-\infty}^{\infty} C_{k}$.

Corollary. For log-concave $c(t):(-\infty, \infty) \rightarrow[0, \infty)$ with $c(0)$ largest, with the same notation except that now $n(\varepsilon)$ and $m(\varepsilon)$ are the larger and smaller solutions of $c(t)=\varepsilon c(0)$

$$
\int_{m(\varepsilon)}^{n(\varepsilon)} c(t) d t \geqslant(1-2 \varepsilon) \int_{-\infty}^{\infty} c(t) d t .
$$

REMARK. In fact the Corollary holds with $\varepsilon$ in place of $2 \varepsilon$ on the right.

Proof of Lemma 3.3. The worst case is when $C_{k}=\varepsilon^{k /(1+n(\varepsilon))} C_{0}$ for $k>0$ and $C_{k}=\varepsilon^{-k /(1+m(\varepsilon))} C_{0}$ for $k<0$. In that case

$$
\sum_{-\infty}^{\infty} C_{k}=C_{0}\left(\frac{1}{1-C_{1}}+\frac{1}{1-C_{-1}}-1\right)
$$

while

$$
\sum_{m(\varepsilon)}^{n(\varepsilon)} C_{k}=\sum_{-\infty}^{\infty} C_{k}-\frac{\varepsilon C_{0}}{1-C_{1}}-\frac{\varepsilon C_{0}}{1-C_{-1}},
$$

and the smaller sum is clearly greater than $(1-2 \varepsilon) \sum_{-\infty}^{\infty} C_{k}$.

Now the terms of (3.13) and (3.14) are, apart from the $\left(1+O\left(\log ^{2} n / n\right)\right)$ factor, a log-concave sequence. The ratio of consecutive terms for $n$ near $\sqrt{u}$ is $\sim \log y$, while for $n$ near $u-\sqrt{u}$ it is less than $\log x / e^{\sqrt{u}}$. Since $\log y \geqslant \log ^{1 / 5} x$, and since $u \geqslant$ $\sqrt{\log x} / R$, in both (3.13) and (3.14) the contribution of the tails is $O(1 / \sqrt{\log x})$ of the whole.

Now let

$$
j_{n}(x, y)=\frac{x \log ^{n-1} x}{(n-1) ! n !}\left(1-\frac{n}{u}\right)^{n-1}\left(1-\frac{n}{\log x-n \log y+n}\right),
$$

and let

$$
L(x, y)=\sum_{n=1}^{[u]} j_{n}(x, y), \quad M(x, y)=\sum_{[\sqrt{u}]}^{[u-\sqrt{u}]} j_{n}(x, y) .
$$

As above, and again using Lemma 3.3, $M(x, y)=L(x, y)(1+O(1 / \sqrt{\log x}))$. Also, $M(x, y)=A(x, y)(1+O(1 / \sqrt{\log x}))$ since between $\sqrt{u}$ and $u-\sqrt{u}$ the terms of $M(x, y)$ and the terms in (3.13) and (3.14) all agree to within a factor of $1+O(1 / \sqrt{\log x})$, and since these terms comprise all but $O(1 / \sqrt{\log x})$ of the whole in each case.

To estimate $L(x, y)$ we introduce a function $f(s)$ so that $x e^{f(u)} \sim j_{n}(x, y)$, and consider $x \int_{0}^{u} e^{f(s)} d s$. We take this $f(s)$ :

$$
f(s)=-\log (2 \pi)-2 s \log s+2 s+(s-1)(\log \log x+\log (1-s / u)) .
$$


Then for $\varepsilon>0$, and $\sqrt{u} \leqslant n \leqslant(1-\varepsilon) u$,

$$
e^{f(n)}=\left(1+O_{\varepsilon}(1 / \sqrt{\log x})\right) j_{n}(x, y) .
$$

Now $f(s)$ is concave. We shall later show that for each $R$ there exists $\varepsilon=\varepsilon(R)$ such that for $r \leqslant R$, with $c(t)=e^{f(t)}$,

$$
\sqrt{u} \leqslant m\left(\log ^{-1 / 2} x\right)<n\left(\log ^{-1 / 2} x\right) \leqslant(1-\varepsilon(R)) u,
$$

in the notation of Lemma 3.3. Consequently,

$$
\begin{aligned}
& \text { All of } L(x, y), M(x, y), \sum_{\sqrt{u}<n<u(1-\varepsilon)} j_{n}(x, y), \sum_{\sqrt{u}<n<u(1-\varepsilon)} e^{f(n)} \\
& \text { and } \Sigma_{n<u} e^{f(n)} \text { are equal to within factors of } 1+O(1 / \sqrt{\log x}) .
\end{aligned}
$$

We then show that $x \Sigma_{n<u} e^{f(n)}=x \int_{0}^{u} e^{f(s)} d s\left(1+O\left(\log ^{-1 / 4} x\right)\right)$, and evaluate this integral to an accuracy of $1+O\left(\log ^{-1 / 4} x\right)$ to obtain Theorem 2 and the Corollaries.

None of this to come is all that deep or complex; it is standard calculus and some lengthy but routine parts are left to the reader.

4. Calculus. Although $x$ and $y$ do not appear explicitly in ' $f(s)$ ', strictly speaking we should write $f(s, x, y)$. This said, we have

$$
f^{\prime}(s)=-2 \log s+\log \log x+\log (1-s / u)-\frac{s-1}{u-s}
$$

and

$$
f^{\prime \prime}(s)=-\left(\frac{2}{s}+\frac{1}{u-s}+\frac{u-1}{(u-s)^{2}}\right) .
$$

Let $S=S(x, y)$ be the unique solution of

$$
-2 \log S+\log \log x+\log (1-S / u)-(S-1) /(u-S)=0, \quad 0<S<u \text {. }
$$

Now recall that $r=\sqrt{\log x} / u$ and that $r \leqslant R$.

LEMMA 4.1. For all $R>0$ there exists $C(R)>0$ such that if $0<r \leqslant R$ and $u \geqslant C(R)$ then $(3 \log (e+r))(u-S) \geqslant u$.

Proof. For $s<S, f^{\prime}>0$ while for $s>S, f^{\prime}<0$. Let $s_{1}=u\left(1-(3 \log (e+r))^{-1}\right)$. Then

$$
\begin{aligned}
f^{\prime}\left(s_{1}\right)= & -2 \log u-2 \log \left(1-(3 \log (e+r))^{-1}\right)+\log \log x-\log 3 \\
& -\log \log (e+r)+3 \log (e+r) / u-3 \log (e+r)+1 \\
< & -\log (e+r)-2 \log (2 / 3)-\log 3+3 \log (e+r) / u+1 \\
< & \log 3-2 \log 2+1-(1-3 / u)=\log (3 / 4)+3 / u \\
< & 0 \text { for } u>3 / \log (4 / 3) .
\end{aligned}
$$

Thus $f^{\prime}\left(s_{1}\right)<0$ so $s_{1}>S$, which proves the lemma with $C=3 / \log (4 / 3)$.

LEMMA 4.2. For fixed $x, S=S(r)$ is decreasing as a function of $r$ for $r \geqslant 0$, and $\lim _{r \rightarrow 0} S(r)=\sqrt{\log x}$. 
Proof. By definition

$$
-2 \log S+\log \log x+\log (1-S / u)-(S-1) /(u-S)=0 .
$$

Let $t(r)=S(r) / \sqrt{\log x}$. Then

$$
0=-2 \log t+\log (1-r t)-\frac{r t}{1-r t}(1-1 / \sqrt{\log x}) .
$$

Thus $d t / d r=t^{\prime}$ satisfies

$$
\begin{gathered}
-\left(2 / t+2 r /(1-r t)+(r /(1-r t))^{2}(t-1 / \sqrt{\log x})\right) t^{\prime} \\
=t /(1-r t)+(1-r t)^{-2}(t-1 / \sqrt{\log x}),
\end{gathered}
$$

so $t^{\prime}<0$. Inspection of (4.4) shows $\lim _{r \rightarrow 0} t(r)=1$.

With the same notation we also have

$$
t(r) \geqslant 1 /(3+3 r) \text { for } r>0 .
$$

Proof. The right side of (4.4) is decreasing in $t$ for fixed $r$, and is positive when $t=(3+3 r)^{-1}$. Thus $t(r)>1 /(3+3 r)$.

In particular, $t(r) \geqslant 1 /(3+3 R)$ for $0<r \leqslant R$, and so $S(r) \geqslant K \sqrt{\log x}$ for $0<r \leqslant R$, with $K=1 /(3+3 R)$ constant. From Lemma 4.1 and from $0<S(r)<u$, it follows that $1 / S, 1 /(u-S)$ and $u /(u-S)^{2}$ are all $O(1 / \sqrt{\log x})$ uniformly in $r \leqslant R$ as $x \rightarrow \infty$. Thus

$$
\left|f^{\prime \prime}(S)\right|=O(1 / \sqrt{\log x}) \text { uniformly in } r \leqslant R \text {. }
$$

LEMMA 4.3. Uniformly in $0<r \leqslant R$ and in $h,|h| \leqslant(S(r))^{2 / 3}$,

$$
\left|f^{\prime \prime}(S)-f^{\prime \prime}(S+h)\right|=O(|h| / \log x)
$$

and

$$
f(S+h)=f(S)+\frac{1}{2} h^{2} f^{\prime \prime}(S)+O\left(|h|^{3} / \log x\right) .
$$

Proof. The second assertion is a direct consequence of the first. The first follows from the calculation

$$
\begin{aligned}
f^{\prime \prime}(S+h)-f^{\prime \prime}(S) & =\left(\frac{2}{S+h}-\frac{2}{S}\right)+\left(\frac{1}{u-S-h}-\frac{1}{u-S}\right) \\
+ & (u-1)\left(\frac{1}{(u-S-h)^{2}}-\frac{1}{(u-S)^{2}}\right) \\
= & -\frac{2 h}{S(S+h)}+\frac{h}{(u-S)(u-S-h)}+\frac{2(u-1) h}{(u-S)(u-S-h)^{2}} \\
& -\frac{h^{2}(u-1)}{(u-s)(u-S-h)^{2}} .
\end{aligned}
$$

Now from (4.5) and Lemma 4.1, respectively, $1 / S=O(1 / u)$ and $1 /(u-S)=$ $O(1 / u)$. Thus the last expression for $f^{\prime \prime}(S+h)-f^{\prime \prime}(S)$ is

$$
h\left\{O\left(1 / u^{2}\right)+O\left(1 / u^{2}\right)+O\left(1 / u^{2}\right)+O\left(u^{2 / 3} / u^{3}\right)\right\}=h \cdot O\left(1 / u^{2}\right) .
$$


Since $u=\log x / \log y$ and $r=\log y / \sqrt{\log x} \leqslant R, 1 / u^{2}=O(1 / \log x)$. Thus

$$
\left|f^{\prime \prime}(S)-f^{\prime \prime}(S+h)\right|=O(|h| / \log x)
$$

as claimed.

Now let $g(s)=e^{f(s)}$. Then $g(s)$ is log-concave with its maximum at $S$. Heuristically, in $\int_{0}^{u} g(s) d s$ most of the mass is found near $S$. Near $S$ though, $g(s)$ is approximately normal, and the approximation has a well-known integral.

To prove the claims of (3.17) and the following paragraph, we first note that

$$
\left|\sum_{n<u} e^{f(n)}-\int_{0}^{u} e^{f(s)} d s\right| \leqslant 2 e^{f(S)} .
$$

Next, let $\bar{m}$ and $\bar{n}$ be the (real) solutions less and more than $r$, respectively, of $f(\bar{m})=f(\bar{n})=f(S)-\frac{1}{2} \log \log x$.

By Lemma 3.3,

$$
\int_{0}^{u} g(s) d s=\int_{\bar{m}}^{\bar{n}} g(s) d s\left(1+O\left(\log ^{-1 / 2} x\right)\right) .
$$

We now claim that $|\bar{m}-S| \leqslant S^{3 / 5},|\bar{n}-S| \leqslant S^{3 / 5}$. For if $h= \pm S^{3 / 5}$ then

$$
\begin{aligned}
f(S+h) & =f(S)+\frac{1}{2} h^{2} f^{\prime \prime}(S)+O\left(|h|^{3} / \log x\right) \\
& \leqslant f(S)-h^{2} / S+O\left(\log ^{-1 / 10} x\right) \\
& \leqslant f(S)-S^{1 / 5}+O\left(\log ^{-1 / 10} x\right) \\
& \leqslant f(S)-\log \log x \quad \text { for large } s, \text { since } S \geqslant K \sqrt{\log x} .
\end{aligned}
$$

Now a little calculation (left to the reader) from Lemma 4.1 shows $\bar{n} \leqslant\left(1-\frac{1}{2} K\right) u$, and another from $\log y \geqslant \log { }^{1 / 5} x, r=\log y / \sqrt{\log x}, S \geqslant \sqrt{\log x} /(3+3 r)$ and $u=\sqrt{\log x} / r$ shows $\bar{m} \geqslant S-S^{3 / 5} \geqslant \sqrt{u}$ for large $x$. This completes the proof of (3.17).

Now

$$
\int_{\bar{m}}^{\bar{n}} g(s) d s=e^{f(s)} \int_{\bar{m}}^{\bar{n}} \exp \left\{\frac{1}{2}(s-S)^{2} f^{\prime \prime}(S)+O\left((s-S)^{3} / \log x\right)\right\} d s,
$$

and

$$
\begin{aligned}
& \int_{\bar{m}}^{\bar{n}}\left|g(s)-\exp \left\{f(S)+\frac{1}{2}(s-S)^{2} f^{\prime \prime}(S)\right\}\right| d s \\
& \quad=O \int_{\bar{m}}^{\bar{n}} \exp \left(f(S)+\frac{1}{2}(s-S)^{2} f^{\prime \prime}(S)\left((s-S)^{3} / \log x\right)\right) d s
\end{aligned}
$$

because $\left|(s-S)^{3} / \log x\right| \leqslant \log ^{-1 / 10} x$ for $\bar{m} \leqslant s \leqslant \bar{n}$. We calculate this last integral as

$$
\begin{aligned}
& O\left(\log ^{-1} x\right) \int_{-\infty}^{\infty} \exp \left(f(S)+\frac{1}{2} v^{2} f^{\prime \prime}(S)\right) v^{3} d v \\
& =O\left(e^{f(S)} / \log x\right) \int_{-\infty}^{\infty} v^{3} \exp \left(\frac{1}{2} v^{2} f^{\prime \prime}(S)\right) d v \\
& =O\left(e^{f(S)}\right) \quad \text { since } f^{\prime \prime}(S)<-\frac{2}{S}<-\frac{\text { const }}{\sqrt{\log x}}
\end{aligned}
$$


On the other hand,

$$
\begin{aligned}
\int_{\bar{m}}^{\bar{n}} \exp \left(f(S)+\frac{1}{2}(s-S)^{2} f^{\prime \prime}(S)\right) d s \\
\quad=\int_{-\infty}^{\infty} \exp \left(f(S)+\frac{1}{2} v^{2} f^{\prime \prime}(S)\right) d v\left(1+O\left(\log ^{-1 / 2} x\right)\right)
\end{aligned}
$$

by Lemma 3.3, and this equals $\sqrt{2 \pi}\left|f^{\prime \prime}(S)\right|^{-1 / 2} e^{f(S)}$.

Since $\left|f^{\prime \prime}(S)\right|>1 / S>1 / \sqrt{\log x}$,

$$
e^{f(S)}=O\left(\log ^{1 / 4} x\right) \int_{\bar{m}}^{\bar{n}} \exp \left(f(S)+\frac{1}{2}(s-S)^{2} f^{\prime \prime}(S)\right) d s .
$$

Putting this together, we get

$$
\int_{\bar{m}}^{\bar{n}} g(s) d s=\sqrt{2 \pi}\left|f^{\prime \prime}(S)\right|^{-1 / 2} e^{f(S)}\left(1+O\left(\log ^{-1 / 4} x\right)\right)
$$

and

$$
A(x, y)=\sqrt{2 \pi}\left|f^{\prime \prime}(S)\right|^{-1 / 2} e^{f(S)}\left(1+O\left(\log ^{-1 / 4} x\right)\right) .
$$

This is essentially Theorem 2 . It remains only to simplify $\left|f^{\prime \prime}(S)\right|^{-1 / 2} e^{f(S)}$. We first note that if $\bar{S}$ is the solution in $0 \leqslant s \leqslant u$ of

$$
2 \log s-\log (1-s / u)+s /(u-s)=\log \log x
$$

$(s$ instead of $s-1$ in $(s-1) /(u-s)$ of $(4.3))$ then

$$
|\bar{S}-S|=O(1)
$$

(left to reader). Since $f^{\prime \prime}(S)=O(1 / \sqrt{\log x})$, it follows by Lemma 4.3 that

$$
|f(\bar{S})-f(S)|=O\left(\log ^{-1 / 2} x\right) .
$$

Let $q=q(r)=\bar{S}(r) / \sqrt{\log x}$ and let $w=w(r)=r q(r)$. Recall $u=\sqrt{\log x} / r$. The original hypotheses for Theorem 2 were that $u \geqslant \log ^{1 / 5} x$ and $r \leqslant R$.

Let

$$
C(r)=\frac{1}{2 \sqrt{\pi}}\left(1-w+\frac{1}{2} w^{2}\right)^{-1 / 2} \text { and } h(r)=2 q(r)+\frac{w(r) q(r)}{1-w(r)}
$$

These definitions agree with those in the introduction, the verification is left to the reader.

A short table of calculus facts for $q(r), w(r)$ and $h(r)$ may be helpful. In every case the derivation is a routine calculation, which we omit.

$$
\begin{aligned}
& d q / d r=-q^{2}(2-w) /\left(2-2 w+w^{2}\right)<0 \\
& d w / d r=2 q(1-w)^{2} /\left(2-2 w+w^{2}\right)>0 \\
& d^{2} q / d r^{2}=2 q^{3}\left(3 w^{2}-8 w+6\right) /\left(2-2 w+w^{2}\right)^{3}>0 \\
& q=1-r+\frac{3}{4} r^{2}+O\left(r^{3}\right), \quad w=r-r^{2}+O\left(r^{3}\right) \\
& h=q\left(1+(1-w)^{-1}\right)=2-r+\frac{1}{2} r^{2}+O\left(r^{3}\right)
\end{aligned}
$$




$$
\begin{aligned}
d h / d r & =-q^{2} /(1-w)<0, \\
d^{2} h / d r^{2} & =q^{3} w /\left((1-w)\left(2-2 w+w^{2}\right)\right)>0 ;
\end{aligned}
$$

$\begin{array}{cccc}r & h(r) & r & h(r) \\ 0 & 2 & & \\ 0.1 & 1.90483 & 0.6 & 1.54523 \\ 0.2 & 1.81865 & 0.7 & 1.49077 \\ 0.3 & 1.74048 & 0.8 & 1.44062 \\ 0.4 & 1.66939 & 0.9 & 1.39428 \\ 0.5 & 1.60456 & 1.0 & 1.35136 \\ 2 & 1.04750 & 1000 & 0.01238 \\ 5 & 0.66283 & 10000 & 0.00167 \\ 10 & 0.43586 & 100000 & 0.000210 \\ 20 & 0.27363 & 1000000 & 0.0000254 \\ 50 & 0.14035 & & \\ 100 & 0.08224 & & \end{array}$

Now since $|f(\bar{S})-f(S)|=O\left(\log ^{-1 / 2} x\right)$ and since $\left|f^{\prime \prime}(\bar{S})-f^{\prime \prime}(S)\right|=O(1 / \log x)$ while $\left|f^{\prime \prime}(S)\right|>1 / \sqrt{\log x}$, the right side of (4.9) is disturbed only by a factor of $1+O(1 / \sqrt{\log x})$ when $S$ is replaced with $\bar{S}$. If we now express the modified right side in terms of $r, q(r), w(r), C(r)$ and $h(r)$ it simplifies to

$$
A(x, y)=C(r) x \log ^{-3 / 4} x \exp (h(r) \sqrt{\log x})\left(1+O\left(\log ^{-1 / 4} x\right)\right)
$$

uniformly in $y$ such that $\log x / \log y>\log ^{1 / 5} x$ and $\log y / \sqrt{\log x} \leqslant R$. This proves Theorem 2.

For the corollaries let $\theta=\log y / \log ^{1 / 4} x$ and assume $\theta=O(1), x \rightarrow \infty$. Then $r=\theta \log ^{-1 / 4} x, C(r)=\left(1+O\left(\log ^{-1 / 4} x\right)\right) / 2 \sqrt{\pi}$, and by the Taylor series expansion of $h(r)$,

$$
\begin{aligned}
\exp (h(r) \sqrt{\log x}) & =\exp \left(2 \sqrt{\log x}-\theta \log ^{1 / 4} x+\frac{1}{2} \theta^{2}+o(1)\right) \\
& \sim y^{-1} \exp \left(2 \sqrt{\log x}+\frac{1}{2} \theta^{2}\right)
\end{aligned}
$$

so that $A(x, y) \sim y^{-1} e^{\theta^{2} / 2} A(x, 1)$.

REMARK. We have not considered $u$ fixed $(u=\log x / \log y)$ as $x \rightarrow \infty$. Ad hoc calculations suggest that for this case, if $n$ is an integer $\geqslant 1$ and if $n<u \leqslant n+1$ then

$$
A\left(x, x^{1 / u}\right) \sim \frac{(u-n)^{n-1}}{n((n-1) !)^{2}} x(\log y)^{n-1}
$$

(conjecture). 


\section{BIBLIOGRAPHY}

1. D. Hensley, The number of factorizations of numbers less than $x$ into factors less than $y$, Trans. Amer. Math. Soc. 275 (1983), 477-496.

2. A. Oppenheim, On an arithmetic function. II, J. London Math. Soc. 2 (1927), 123-130.

3. G. Szekeres and P. Turán, Über das zweite Hauptproblem der "Factorisatio Numerorum", Acta Sci. Math. (Szeged) 6 (1933), 143-154.

Department of Mathematics, Texas A \& M University, College Station, Texas 77843 